\title{
Light Cone wavefunction approach to open heavy flavor dynamics in the QGP
}

\author{
Rishi Sharma ${ }^{a}$ \\ ${ }^{a}$ Group T-2, Theoretical Division, Los Alamos National Laboratory, Los Alamos, NM, 87545, USA
}

\begin{abstract}
We elucidate the role of time scales that determine heavy quark dynamics in the QGP. Quarkantiquark potentials extracted from the lattice are used to demonstrate the existence of open heavy flavor bound-state solutions in the vicinity of the critical temperature, and their light cone wavefunctions are obtained. We use these wavefunctions to calculate the in-medium modification of the heavy quark distribution and decay probabilities. For the case of high $p_{T} D$ or $B$ mesons traversing the QGP, we combine the new meson formation and dissociation mechanism with the traditional parton-level charm and beauty quark quenching to obtain predictions for the heavy meson and non-photonic electron suppression in $\mathrm{Cu}+\mathrm{Cu}$ and $\mathrm{Pb}+\mathrm{Pb}$ collisions at $\mathrm{RHIC}$ and the LHC, respectively.
\end{abstract}

\section{Inadequacy of partonic level energy loss for heavy flavor suppression}

It is well known that partonic level energy loss of $c$ and $b$ quarks in the QGP is insufficient to explain the large suppression $\left(R_{A A}\right)$ of non-photonic $e^{+}+e^{-}$measured at RHIC [1]. To correctly assess the discrepancy between the expected heavy quark quenching and the measured $R_{A A}$ [2], improved treatment of cold nuclear matter effects [3, 4] is necessary. It is, therefore, important to establish the role of (i) shadowing, (ii) Cronin effect and (iii) cold nuclear matter energy loss in the analysis of open heavy flavor suppression. Our results [5] bring the treatment of cold nuclear matter effects for open heavy flavor production on par with their implementation in the study of light hadron and direct photon final states [6].

We now turn to the final-state quark and gluon dynamics. The standard paradigm for jet quenching is to consider the energy lost by a parton traversing through the deconfined medium. This parton then fragments outside the QGP. We perform the energy loss calculation in the framework of the GLV approach [7]. The properties of the soft gluon-dominated matter is constrained by the experimentally measured charged particle pseudorapidity density [5].

The results for $R_{A A}$ of $\pi^{0}$ calculated thus, describe the experimental data beautifully. On the other hand the experimental results on non-photonic electrons [2], $R_{A A}^{e^{ \pm}} \sim R_{A A}^{\pi^{0}}$ for $p_{T}>5 \mathrm{GeV}$, are in clear contradiction with the small quenching of $B$ mesons that give an increasingly important ( $\geq 50 \%$ ) contribution to non-photonic $e^{+}+e^{-}$in this region [8, 9]. Cold nuclear matter effects increase the discrepancy between the results of partonic level quenching and experiments [5].

This discrepancy forces a check of our basic assumptions. Whether or not an energetic quark traversing the QGP forms a (possibly short-lived) hadronic state in the plasma depends on whether the formation time of the hadron, $\tau_{\text {form }}$ [8], is less or greater than the plasma life time. The small pion mass ensures that the parent light quarks and gluons fragment outside of the QGP 
in accord with the traditional picture of jet quenching. The large $D$ and $B$ meson mass, however, implies that charm and beauty quarks will fragment inside the hot medium: $\tau_{\text {form }} \propto 1 / m_{h}^{2}$. Consequently, the competition between heavy meson dissociation and the $c$ and $b$ quark decay in the QGP is a likely physics mechanism that may naturally lead to attenuation of the beauty cross section as large as that for charm [8].

\section{Can a $D$ or $B$ meson exist in the QGP?}

To understand if a $D$ or $B$ meson can exist at rest and in thermal equilibrium in a QGP, and to understand how the parton distribution functions (PDFs) and fragmentation functions (FFs) are modified in a thermal medium, we solve the Dirac equation for the light quark in a thermally modified Cornell potential. Whether such thermal effects do indeed take place for the relevant case of an energetic meson moving through the medium, depends on whether the time taken to equilibrate this meson is larger or smaller than the time taken to traverse the QGP. We will address this question in the next section, and for this section consider the case of a $D$ or $B$ meson in equilibrium with the medium.

Lattice QCD results for the free energy of a system at temperature $T$ containing two infinitely heavy quarks separated by a distance $r$ [10] have been used to extract the static potential between these quarks. This potential has been employed previously to study the fate of heavy-heavy bound states in the QGP formed at temperatures above $T_{c}$ [11]. We perform a similar study to look at the possibility of the existence of heavy-light mesons in the QGP by looking for bound state solutions for the Dirac equation describing the motion of the light quark in the potential set up by the heavy quark. The temperature up to which bound states exist depends sensitively upon the details of the potential chosen and the effective in-medium mass of the light quark. Here we quote results (Table. 1) for a relatively strongly binding potential and take the in-medium mass of the light quark to be the $m(T) / \sqrt{2}$. More details are given elsewhere [5]. We find that $D$ - and $B$-meson bound-state solutions persist above $T_{c}$. Their small binding energy and large radius, however, will greatly facilitate their subsequent dissolution in the presence of interactions.

We can use the bound state wavefunctions obtained to calculate the thermally modified PDFs and FFs. This calculation is most conveniently done using the wavefunctions in the light cone form [12]. The first results for how the PDFs and FFs change with temperature are in [5].

\section{Application to heavy meson production in heavy ion collisions}

Heavy flavor dynamics in hot QCD matter critically depends on the time scales involved in the underlying reaction. As discussed in the previous section, the dissociation of $D$ and $B$ mesons in the vicinity of $T_{c}$ can be facilitated by their small binding energy. Whether such thermal effects

\begin{tabular}{c|c|c|c|c|c|c|c|c|c}
$T$ & 0 & $0.2 T_{c}$ & $0.4 T_{c}$ & $0.6 T_{c}$ & $0.8 T_{c}$ & $1.0 T_{c}$ & $1.2 T_{c}$ & $1.4 T_{c}$ & $1.6 T_{c}$ \\
\hline$T(\mathrm{GeV})$ & 0 & 0.038 & 0.077 & 0.115 & 0.154 & 0.211 & 0.230 & 0.269 & 0.307 \\
$E_{b}(\mathrm{GeV})$ & 0.730 & 0.733 & 0.611 & 0.256 & 0.098 & 0.043 & 0.031 & 0.017 & 0.009 \\
$\sqrt{\left\langle r^{2}\right\rangle} \mathrm{fm}$ & 0.468 & 0.466 & 0.464 & 0.501 & 0.632 & 0.785 & 0.970 & 1.263 & 1.636
\end{tabular}

Table 1: Properties of the bound $D$ - and $B$-meson bound-state solutions taking the effective light quark mass to be $m(T) / \sqrt{2}$. These solutions persist up to temperatures $\approx 1.6 T_{c}$. 
Figure 1: (Left.) Suppression of $D$ and $B$ hadron production from meson dissociation and heavy quark quenching in central $\mathrm{Au}+\mathrm{Au}$ (top panel) and $\mathrm{Cu}+\mathrm{Cu}$ collisions (bottom panel) at $\sqrt{s_{N N}}=200 \mathrm{GeV}$ at RHIC. (Right.) Nuclear modification for the single non-photonic electrons in central $\mathrm{Au}+\mathrm{Au}$ and $\mathrm{Cu}+\mathrm{Cu}$ collisions at RHIC. Data is from PHENIX and STAR [2] collaborations.
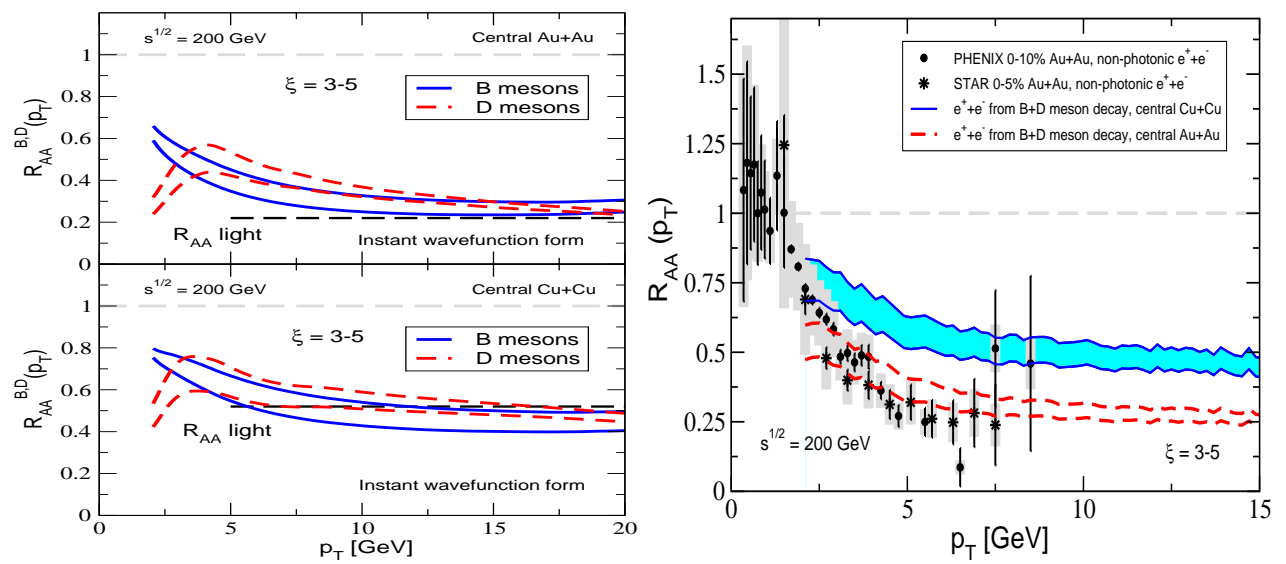

take place in practice, however, depends on the time they need to develop. We can roughly estimate this time by boosting the expanded size of the hadron, $\approx 2 \sqrt{\left\langle r^{2}\right\rangle}$ from Table 1 , by the $\gamma$ factor. When compared to $\tau_{\text {form }}$ this time is large and suggests that the fragmentation component of the heavy meson dynamics in heavy ion collisions may not be affected by the QGP. In what follows we will study this "instant wavefunction limit".

A meson that is formed and propagates inside the medium will undergo collisional broadening and dissociation [8, 13]. Following the diagrammatic calculation described in [8] one can calculate the dissociation time $\tau_{\text {diss }}$ for a meson of transverse momentum $p_{T}$.

Employing the rates, $\tau_{\text {diss }}$ and $\tau_{\text {form }}$, the concurrent processes of $c$ and $b$ quark fragmentation and $D$ and $B$ meson dissociation are described by rate equations [8] describing the time evolution of quark and meson "densities" $\left(f^{Q}\left(p_{T}, t\right)=\frac{d \sigma^{Q}(t)}{d y d^{2} p_{T}}\right.$ and $f^{H}\left(p_{T}, y, t\right)=\frac{d \sigma^{H}(t)}{d y d^{2} p_{T}}$ respectively). At present, there is no reliable way of incorporating the fluctuations in partonic energy loss in rate or transport equations. Therefore, we include the early-time heavy quark inelastic scattering effects approximately as a quenched initial condition, $f^{Q}\left(p_{T}, 0\right)=\frac{d \sigma^{Q, \text { Quench }}}{d y d^{2} p_{T}}, f^{H}\left(p_{T}, 0\right)=0$. Here, the attenuated partonic spectrum $\frac{d \sigma^{Q, \text { Quench }}}{d y d^{2} p_{T}}$ is calculated differentially versus $p_{T}$ using partonic level quenching. The relevant mean quenching time - the time that the physical system of interest spends in a quark state - can be estimated from an analytic solution for $f^{Q}\left(p_{T}, t\right), f^{H}\left(p_{T}, t\right)[5]$.

We integrate numerically the above set of coupled ordinary differential equations and use the same initial soft gluon rapidity density $d N^{g} / d y$ as in the simulations of $\pi^{0}$ quenching. The corresponding suppression of open heavy flavor in $\sqrt{s_{N N}}=200 \mathrm{GeV}$ central $\mathrm{Au}+\mathrm{Au}$ and $\mathrm{Cu}+\mathrm{Cu}$ collisions at RHIC is shown in the top and bottom panels on the left in Fig. 11 respectively. For $D$ mesons the enhancement from Cronin effect is clearly visible around $p_{T} \sim 4 \mathrm{GeV}$. In both gold and copper reactions at RHIC the suppression $R_{A A}^{B} \approx R_{A A}^{D}$ for $p_{T}>4 \mathrm{GeV}$ and these approach the quenching of light hadrons for $p_{T}>10 \mathrm{GeV}$. Future vertex detector upgrades at RHIC will ensure direct and separate measurements of the $D$ and $B$ mesons and can be compared directly with our results. We also give $R_{A A}$ for non-photonic electrons calculated using semi-leptonic decays of charm and beauty hadrons using PYTHIA [14]. Results for the suppression of open 
Figure 2: Suppression of $D$ and $B$ meson production in central $\mathrm{Pb}+\mathrm{Pb}$ collisions at $\sqrt{s_{N N}}=5500 \mathrm{GeV}$ at the LHC in two different scenarios. The top panel shows the quenching of heavy hadrons only due to partonic energy loss. The bottom panel gives $R_{A A}$ for $D$ s and $B$ s with partonic energy loss as well as collisional dissociation of heavy meson. Insert shows the corresponding attenuation of non-photonic electrons in a limited $p_{T}$ range.

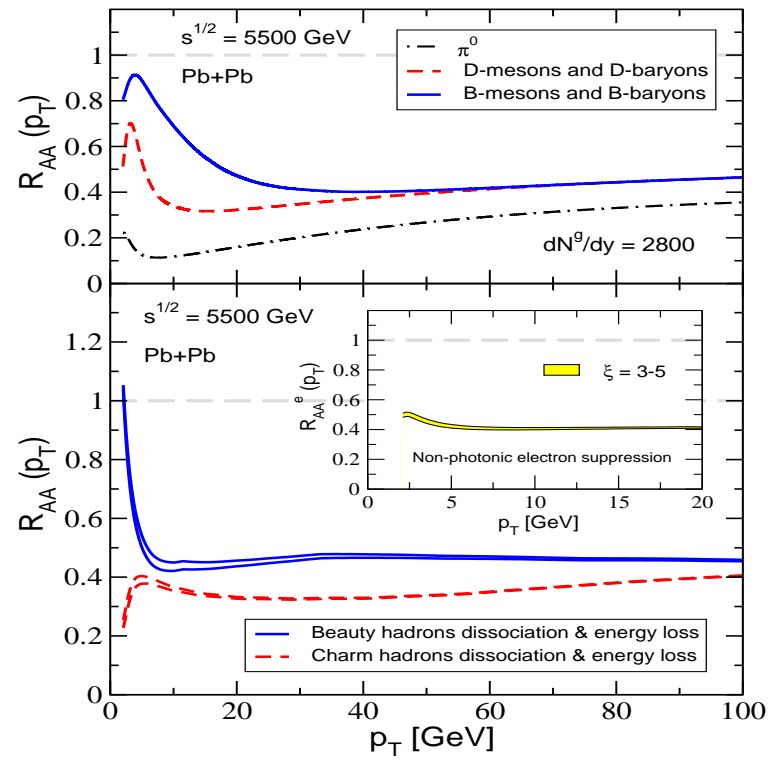

heavy flavor final states in central $\mathrm{Pb}+\mathrm{Pb}$ collisions at $\sqrt{s_{N N}}=5500 \mathrm{GeV}$ at the LHC taking $d N^{g} / d y=2800$ are presented in Fig. 2] $R_{A A}$ for non-photonic electrons is shown in the insert.

\section{Acknowledgments}

We would like to thank Ivan Vitev and Benwei Zhang for collaboration on the work and for reading the manuscript. This research is supported by the US Department of Energy, Office of Science, under Contract No. DE-AC52- 06NA25396 and by the LDRD program at LANL.

\section{References}

[1] S. Wicks, W. Horowitz, M. Djordjevic and M. Gyulassy, Nucl. Phys. A 783, 493 (2007).

[2] A. Adare et al. [PHENIX], and B. I. Abelev et al. [STAR], Phys. Rev. Lett. 98, 172301 and 192301 (2007)

[3] I. Vitev, Phys. Lett. B 562, 36 (2003) and Phys. Rev. C 75, 064906 (2007).

[4] I. Vitev, T. Goldman, M. B. Johnson and J. W. Qiu, Phys. Rev. D 74, 054010 (2006).

[5] R. Sharma, I. Vitev and B. W. Zhang, arXiv:0904.0032 [nucl-th]; v2 unpublished

[6] I. Vitev and B. W. Zhang, Phys. Lett. B 669, 337 (2008).

[7] M. Gyulassy, I. Vitev, X. N. Wang and B. W. Zhang, arXiv:nucl-th/0302077

[8] A. Adil and I. Vitev, Phys. Lett. B 649, 139 (2007).

[9] G. Wang [STAR Collaboration], J. Phys. G: Nucl. Part. Phys. 35104107 (2008).

[10] O. Kaczmarek and F. Zantow, Phys. Rev. D 71, 114510 (2005).

[11] A. Mocsy and P. Petreczky, and Phys. Rev. D 77, 014501 (2008), and Phys. Rev. Lett. 99, 211602 (2007).

[12] S. J. Brodsky, H. C. Pauli and S. S. Pinsky, Phys. Rept. 301, 299 (1998).

[13] F. Dominguez and B. Wu, Nucl. Phys. A 818, 246 (2009).

[14] T. Sjostrand, S. Mrenna and P. Skands, JHEP 0605, 026 (2006). 